\title{
Self-calibration algorithm for an IMU in two-wheeled vehicles: design and experimental validation
}

\author{
Simone Gelmini ${ }^{1, *}$, Majda Fouka ${ }^{2}$, Silvia Strada ${ }^{1}$, Mara Tanelli ${ }^{1}$, \\ Sergio Savaresi ${ }^{1}$, and Claudio De Tommasi ${ }^{3}$
}

\begin{abstract}
In this paper an algorithm for the estimation of the three mounting angles (roll, pitch, and yaw) of accelerometers and gyroscopes within Inertial Measurement Units (IMUs) is presented. Such a self-calibration method is focused for telematic boxes (e-Boxes) installed on two-wheeled vehicles, whose IMUs' axes often result not to be aligned with the vehicle reference system. Previous methods for axes calibration commonly use satellite-based radionavigation data to determine the unknown mounting angles. In this paper we propose an energy-efficient alignment procedure which limits the use of geolocation data. The aspects of data selection and real-time implementation of our method are taken particularly into account. The proposed approach is validated and performance are analyzed on experimental data collected with tests performed with a motorcycle equipped with three e-Boxes mounted in different positions and orientations. The analysis of the real measured driving data proves the effectiveness of the approach in aligning the sensors' axes in all three directions.
\end{abstract}

\begin{tabular}{cll}
\multicolumn{2}{c}{ NOTATION } \\
$A^{\prime}$ & Transpose of matrix $A$ \\
$I$ & Identity matrix \\
$c_{\alpha}$ & Cosine of $\alpha$ & \\
$s_{\beta}$ & Sine of $\beta$ & \\
$t_{\gamma}$ & Tangent of $\gamma$ & \\
$g$ & Gravity & $9.81\left[\frac{\mathrm{m}}{\mathrm{s}^{2}}\right]$
\end{tabular}

\section{INTRODUCTION}

Axes calibration is a mandatory operation when inertial sensors mounted within some sort of Electronic Control Unit (ECU) installed on a vehicle must be used for measuring or estimating variables of interest for vehicle control, see e.g., [1], [2], [3], [4]. In fact, inertial sensors are strongly affected by mounting angles, and the correct rotation to be applied in order to recover an alignment which is consistent with the vehicle motion is paramount to obtain sensible data to be employed as representative of the variables of interest, [2], [5], [6]. In general, such procedures are applied to IMUs, which consist of accelerometers and gyroscopes. As additional sensors, which are not object of calibration,

\footnotetext{
${ }^{1}$ Simone Gelmini, Silvia Strada, Mara Tanelli, and Sergio Savaresi are with the Dipartimento di Elettronica, Informazione e Bioingegneria, Politecnico di Milano, Piazza Leonardo da Vinci 32, 20133 Milan, Italy. Email: simone.gelmini, silvia.strada, mara.tanelli, sergio.savaresiepolimi.it

${ }^{2}$ Majda Fouka is with the IBISC LAB, Evry Val d'Essonne University (UEVE), 43 Rue du Pelvoux, 91080 Courcouronnes, France. Email: madjda amina_ aida. fouka@g.enp.edu.dz

${ }^{3}$ Claudio De Tommasi is with AlfaEvolution Technology S.p.A., Via Stalingrado 37 - 40128 Bologna, Italy. Email: claudio.detommasiealfaevolutiontechnology. it

* corresponding author
}

though, Global Navigation Satellite Systems (GNSS) or Global Positioning Systems (GPS) are also present, [7]. In general, the aim of the calibration is to align axes of sensors present in various aftermarket equipments, which may be installed in non-standard positions, and for which the internal alignment of the sensors is also in general not known. In the context of this work, the primary application is the installation of an e-Box for insurance telematics dedicated to two-wheeled vehicles. In two-wheeled vehicles, such a calibration step becomes even more crucial than in cars, as space and vibration constraints often do not allow installations of the needed IMUs in such a way to obtain a nearly natural alignment with the direction of motion, so that the mounting angles can result in being quite significant. To perform such a calibration, some of the existing methods, see e.g., [8], use the GNSS to compare its data to the sensors outputs. This, however, implies that GNSS must be always active and running at its highest possible frequency, so as to match the content of dynamic data. This results in a significantly increase in energy consumption, as an accelerometer/gyroscope pair requires approximately $1 \mathrm{~mA}$ at $3 \mathrm{~V}$, thus an energy of $3 \mathrm{~mW}$, while GNSS consumes $10-20 \mathrm{~mA}$ at $3 \mathrm{~V}$, thus $30-60 \mathrm{~mW}$. In applications where energy consumption is critical, as it is in two-wheeled vehicles, and in general in electric vehicles, algorithms that make sporadic use of the GNSS are to be preferred.

Furthermore, GNSS data are subject to errors (e.g., satellites' signal reflection [7]) which affects the measurement precision. Error is time variant and cannot be predicted a priori. GNSS data reliability may impact on the accuracy of the axes calibration or, eventually, on the solution convergence rate.

In this work, GNSS data is only used to partition the data sets for the initial calibration. Assuming that calibration is performed only at the beginning of the vehicle motion after the e-Box is installed, and possibly re-checked once in a while for robustness, the proposed algorithm ensures an energy efficient approach to the problem.

Overall, the proposed approach is inspired to [6], which is extended to provide practical conditions for data selection and methods for an online implementation. An experimental setup with a Piaggio MP3 scooter has been used to install different e-Boxes in various positions characterized by different initial inclinations, and the performance of the algorithm have been tested on different data sets, proving the effectiveness of the proposed approach.

The structure of the paper is as follows: Section II illus- 
trates the considered problem and presents the experimental setup used in this work. Section IV describes the adopted algorithm and the steps it undertakes to perform the needed axes calibration. Finally Section V thoroughly analyses the experimental tests carried out to assess the performance of the approach, which favorably witness its capability of performing calibration for subsequent use of the accelerations in various applications.

\section{PROBLEM STATEMENT AND EXPERIMENTAL SETUP}

In this paper, a self-calibration algorithm for triaxial IMUs installed on motorcycles is proposed. The aim of the algorithm is to estimate the mounting angles, defined as the angles between the IMU and the vehicle reference system, assumed constant over time. The estimation of these angles makes it possible to virtually rotate the measured accelerations and angular speeds according to the conventional vehicle longitudinal, lateral and vertical axes, denoted as $x_{v}, y_{v}, z_{v}$ respectively.

The experimental setup is composed of telematic e-Boxes, equipped with an IMU with sampling frequency of $400 \mathrm{~Hz}$, and a GNSS unit, the sampling frequency of which is $10 \mathrm{~Hz}$. As shown in Fig. 1, three e-Boxes are installed on the vehicle: two (termed e-Box 1 and e-Box 3) are mounted skewed with respect to the vehicle's reference system, while $e$-Box 2 is mounted underseat on a flat surface, only slightly misaligned with respect to the $x-y$ axes. The three positions have been chosen to represent very different possible mountings that end users may adopt when installing the e-Boxes for telematic data transmission.

Validation data are collected riding a $500 \mathrm{~cm}^{3}$ Piaggio MP3 ABS ASR. Tests are conducted under mixed traffic conditions, both on urban and extra-urban roads.

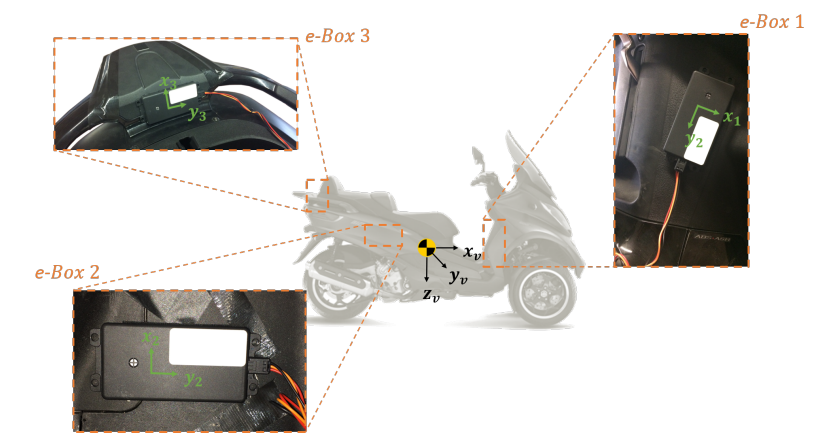

Fig. 1: An overview of the experimental setup used to validate the algorithm.

\section{PRELIMINARIES}

Expressing a vector in a different reference field is a well known problem of linear algebra and studied in many engineering applications, see e.g., [9], [10], [11]. Defining $\mathbf{v}_{1}$ in $\mathbf{X}_{1} \mathbf{Y}_{1} \mathbf{Z}_{1}, \mathbf{v}_{2}$ - the projection of $\mathbf{v}_{1}$ in $\mathbf{X}_{2} \mathbf{Y}_{2} \mathbf{Z}_{2}$ - can be obtained by finding a rotation matrix $R \in \mathbb{R}^{3 \times 3}$ such that

$$
\mathbf{v}_{2}=R \cdot \mathbf{v}_{1}
$$

As illustrated in e.g., [12], [7], matrix $R$ is an orthogonal matrix, which means that it must satisfy

$$
R^{\prime} R=I
$$

where $I$ is the identity matrix. The mathematical relation expressed in (2) imposes six constraints of orthonormality, leaving only three degrees of freedom out of the initial the nine elements of the matrix $R$. Thanks to Euler's Theorem, which states that the general rotation of a rigid frame is equivalent to a rotation around a fixed axis, the three degrees of freedom can be expressed in terms of successive elementary rotations around any combination of $\mathbf{X}, \mathbf{Y}$ or $\mathbf{Z}$. These elementary rotations can be expressed by means of three direction cosine matrices (DCMs):

- rotation about the $\mathrm{x}$-axis through the roll angle $(\varphi)$

$$
\operatorname{DCM}_{\varphi}=\left[\begin{array}{ccc}
1 & 0 & 0 \\
0 & \cos (\varphi) & \sin (\varphi) \\
0 & -\sin (\varphi) & \cos (\varphi)
\end{array}\right]
$$

- a rotation about the $y$-axis through the pitch angle $(\vartheta)$

$$
D C M_{\vartheta}=\left[\begin{array}{ccc}
\cos (\vartheta) & 0 & -\sin (\vartheta) \\
0 & 1 & 0 \\
\sin (\vartheta) & 0 & \cos (\vartheta)
\end{array}\right]
$$

- a rotation about the $\mathrm{z}$-axis through the yaw angle $(\psi)$

$$
D C M_{\psi}=\left[\begin{array}{ccc}
\cos (\psi) & \sin (\psi) & 0 \\
-\sin (\psi) & \cos (\psi) & 0 \\
0 & 0 & 1
\end{array}\right]
$$
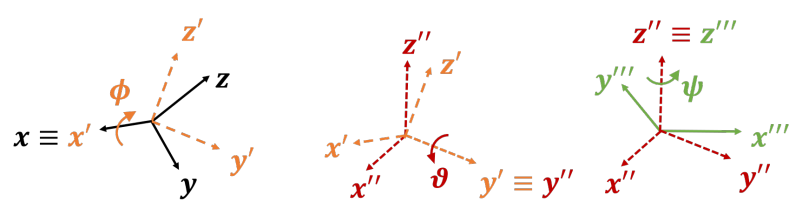

Fig. 2: The alignment of the axes $x, y, z$ to the tern $x^{\prime \prime}, y^{\prime \prime}, z^{\prime \prime}$, following the $\mathbf{X}-\mathbf{Y}-\mathbf{Z}$ convention.

In this paper, we follow the $\mathbf{X}-\mathbf{Y}-\mathbf{Z}$ convention, as illustrated in Fig. 2. This means that our matrix $R$ is obtained multiplying the DCMs in the chosen order, $R=D C M_{\varphi}$. $D C M_{\vartheta} \cdot D C M_{\psi}$. Simplifying the notation with $c_{\alpha}$ denoting $\cos (\alpha), s_{\beta}$ the sine of $\beta$, and $t_{\gamma}$ the tangent of $\gamma$, matrix $R$ becomes

$$
R=\left[\begin{array}{ccc}
c_{\psi} c_{\vartheta} & c_{\vartheta} s_{\psi} & -s_{\vartheta} \\
c_{\psi} s_{\varphi} s_{\vartheta}-c_{\varphi} s_{\psi} & c_{\varphi} c_{\psi}+s_{\varphi} s_{\psi} s_{\vartheta} & c_{\vartheta} s_{\varphi} \\
s_{\varphi} s_{\psi}+c_{\varphi} c_{\psi} s_{\vartheta} & c_{\varphi} s_{\psi} s_{\vartheta}-c_{\psi} s_{\varphi} & c_{\varphi} c_{\vartheta}
\end{array}\right]
$$

Thanks to the orthonormality and orthogonality properties of $R$, it is possible to obtain the transformation from $\mathbf{X}_{2} \mathbf{Y}_{2} \mathbf{Z}_{2}$ to $\mathbf{X}_{1} \mathbf{Y}_{1} \mathbf{Z}_{1}$ by inverting the rotation matrix $R^{-1}=$ $D C M_{-\varphi} \cdot D C M_{-\vartheta} \cdot D C M_{-\psi}$. 


\section{SELF-CALIBRATION ALGORITHM}

In this section, the self-calibration algorithm is presented. Thanks to the mathematical background defined in Section III, the problem of aligning the measurement axes with respect to the vehicle ones can be formulated as the problem of estimating the angles $\varphi, \vartheta, \psi$.

In the proposed algorithm, the estimation of the mounting angles is carried out in two subsequent steps, as illustrated in Fig. 3. First, roll $\varphi$ and pitch $\vartheta$ are estimated aligning the vertical axis with respect to gravity. Then, thanks to the estimated angles, the $x$ and $y$ axes are aligned estimating $\psi$ thanks to appropriate assumptions on the current vehicle longitudinal dynamics.

\section{A. Estimation of $\varphi, \vartheta$}

To estimate $\varphi$ and $\vartheta$, it is assumed the vehicle is driving in a stationary fashion, (i.e., driving at constant speed) on a flat surface, maintaining full contact with the road (i.e., with a vertical acceleration that can be assumed to be negligible). On the contrary of what is assumed in [6], where the authors do not require the vehicle to be in motion, this additional condition constraints the estimate not to be performed when the vehicle is standing still, avoiding the influence of an extra rotation due to the lateral kickstand, a condition not present in four-wheels vehicles but that will often happen in twowheeled ones.

Under these assumptions, the acceleration on the vehicle reference system can be expressed as

$$
a_{v}=\left[\begin{array}{l}
0 \\
0 \\
g
\end{array}\right]
$$

where $g$ is gravity. However, since the telematic box is not aligned with the vehicle's frame, the IMU's acceleration vector $a_{m}$ is rotated by $R$. Defining $a_{x_{m}}, a_{y_{m}}$, and $a_{z_{m}}$ as the accelerations measured by the IMU, $a_{m}$ can be expressed as

$$
a_{m}=R \cdot a_{v}=\left[\begin{array}{c}
-s_{\vartheta} g \\
c_{\vartheta} s_{\varphi} g \\
c_{\varphi} c_{\vartheta} g
\end{array}\right]=\left[\begin{array}{l}
a_{x_{m}} \\
a_{y_{m}} \\
a_{z_{m}}
\end{array}\right] .
$$

Clearly, if the IMU is not aligned, gravity influences all three axes and this effect is only function of $\varphi$ and $\vartheta$. To estimate these two angles, a quadratic cost function $J_{g}$ is defined as the sum of the squared error between $a_{v}$ and $a_{m}$ as

$$
\begin{aligned}
J_{g}(\varphi, \vartheta)= & \left(a_{x_{m}}+s_{\vartheta} g\right)^{2}+\left(a_{y_{m}}-c_{\vartheta} s_{\varphi} g\right)^{2}+ \\
& \left(a_{z_{m}}-c_{\varphi} c_{\vartheta} g\right)^{2} .
\end{aligned}
$$

The estimates of pitch $\widehat{\vartheta}$ and roll $\widehat{\varphi}$ are obtained minimizing (6)

$$
\left[\begin{array}{l}
\widehat{\varphi} \\
\widehat{\vartheta}
\end{array}\right]=\arg \min _{\varphi, \vartheta} J_{g}(\varphi, \vartheta) .
$$

The optimization problem can be solved in closed form and the solution can be found as

$$
\left\{\begin{array} { l l } 
{ \frac { \partial J _ { g } ( \varphi , \vartheta ) } { \partial \vartheta } } & { = 0 } \\
{ \frac { \partial J _ { g } ( \varphi , \vartheta ) } { \partial \varphi } } & { = 0 }
\end{array} \rightarrow \left\{\begin{array}{l}
\varphi=\operatorname{atan} 2\left(a_{y_{m}}, a_{z_{m}}\right) \\
\vartheta=\operatorname{atan} 2\left(-a_{x_{m}}, s_{\varphi} a_{y_{m}}+c_{\varphi} a_{z_{m}}\right)
\end{array}\right.\right.
$$

Before moving to the second part of the algorithm, few considerations are addressed:

- thanks to the result obtained in (8), at each new sample we obtain the value of the two angles which minimize (6), according to the assumptions made in (4). Because of sensor's noise and drifts, the estimate is never constant. To remove these undesired effects, since the mounting angles are assumed not to be changing over time, the estimated values are averaged;

- the estimation of $\varphi$ and $\vartheta$ strongly depends on the assumptions made on the vehicle motion, used to collect that data based on which the estimation is made. Such a data selection step is done by checking that the acceleration norm (i.e., $\|a\|=\sqrt{a_{x_{m}}^{2}+a_{y_{m}}^{2}+a_{z_{m}}^{2}}$ ) is approximately equal to gravity (i.e., $|a| \approx g$ ), as this implies a quasi-stationary motion, with almost constant speed;

- during the averaging process, significant variations of the two angles may be due to variations of road slope, banking or any tilting action performed by the driver. To avoid that the estimates are biased, any detected outlier is removed by resetting the average process.

\section{B. Estimation of $\psi$}

Once the pitch and roll mounting angles are determined, they can be used to estimate $\psi$. To estimate the yaw, it is assumed the vehicle moves on a flat road surface, experiencing only a forward acceleration. Lateral acceleration is assumed negligible (i.e., $a_{y} \approx 0$ ) and the vehicle is assumed always in contact with the road surface $\left(a_{z} \approx 0\right)$. These assumptions can be mathematically expressed in the vehicle reference as

$$
a_{v}=\left[\begin{array}{c}
a_{x_{v}} \\
0 \\
g
\end{array}\right] \text {. }
$$

In this case, the measured acceleration vector becomes

$$
a_{m}=R \cdot a_{v}=\left[\begin{array}{c}
c_{\psi} c_{\vartheta} a_{x_{v}}-s_{\vartheta} g \\
\left(c_{\psi} s_{\varphi} s_{\vartheta}-c_{\varphi} s_{\psi}\right) a_{x_{v}}+c_{\vartheta} s_{\varphi} g \\
\left(s_{\varphi} s_{\psi}+c_{\varphi} c_{\psi} s_{\vartheta}\right) a_{x_{v}}+c_{\varphi} c_{\vartheta} g
\end{array}\right],
$$

in which $\varphi$ and $\vartheta$ can be substituted with the correspondent estimates.

By substituting $a_{m}$ with its components and factorizing $a_{x_{v}}$ and $g$, Equation (10) becomes

$$
\left[\begin{array}{l}
a_{x_{m}} \\
a_{y_{m}} \\
a_{z_{m}}
\end{array}\right]=\left[\begin{array}{cc}
c_{\psi} c_{\vartheta} & -s_{\vartheta} \\
\left(c_{\psi} s_{\varphi} s_{\vartheta}-c_{\varphi} s_{\psi}\right) & c_{\vartheta} s_{\varphi} \\
\left(s_{\varphi} s_{\psi}+c_{\varphi} c_{\psi} s_{\vartheta}\right) & c_{\varphi} c_{\vartheta}
\end{array}\right]\left[\begin{array}{c}
a_{x_{v}} \\
g
\end{array}\right] .
$$

Thanks to the estimated pitch and roll angles, gravity can be compensated on the measured angles, as 


$$
\left[\begin{array}{c}
a_{x}+s_{\vartheta} g \\
a_{y}-c_{\vartheta} s_{\varphi} g \\
a_{z}-c_{\varphi} c_{\vartheta} g
\end{array}\right]=\left[\begin{array}{c}
c_{\psi} c_{\vartheta} \\
\left(c_{\psi} s_{\varphi} s_{\vartheta}-c_{\varphi} s_{\psi}\right) \\
\left(s_{\varphi} s_{\psi}+c_{\varphi} c_{\psi} s_{\vartheta}\right)
\end{array}\right] a_{x_{v}} .
$$

By factorizing $c_{\psi}, s_{\psi}$, we obtain

$$
\underbrace{\left[\begin{array}{c}
\frac{a_{x}+s_{\vartheta} g}{c_{\vartheta}} \\
\frac{a_{y}-c_{\vartheta} s_{\varphi} g}{-c_{\varphi}} \\
\frac{a_{z}-c_{\varphi} c_{\vartheta} g}{s_{\varphi}}
\end{array}\right]}_{y}=\underbrace{\left[\begin{array}{cc}
1 & 0 \\
-t_{\varphi} s_{\vartheta} & 1 \\
t_{\varphi}^{-1} s_{\vartheta} & 1
\end{array}\right]}_{\phi} \underbrace{\left[\begin{array}{c}
c_{\psi} a_{x_{v}} \\
s_{\psi} a_{x_{v}}
\end{array}\right]}_{\theta}
$$

which can now be rewritten as a least squares (LS) problem $y=\phi \cdot \theta$.

Once vector $\theta$ is estimated, the ratio of the two parameters is equal to

$$
\frac{\theta(1)}{\theta(2)}=\frac{c_{\psi} a_{x_{v}}}{s_{\psi} a_{x_{v}}}=\frac{c_{\psi}}{s_{\psi}}
$$

which is the tangent of $\psi$. The estimate of $\psi$ is then obtained by computing the inverse tangent of the parameters ratio

$$
\psi=\operatorname{atan} 2(\theta(2), \theta(1)) .
$$

The assumptions made for estimating $\psi$ are very different than those made for estimating $\varphi$ and $\vartheta$. In this context, the motorbike is assumed to accelerate and brake intensely, a dual condition with respect to the previous one. Thus, the data discarded in the estimate of pitch and roll can in principle be used to estimate $\psi$.

The estimation of $\varphi$ and $\vartheta$, including the data selection phase, is summarized in Fig. 3.

\section{EXPERIMENTAL RESULTS}

The proposed algorithm is tested against experimental data collected over few hours of driving, divided in three te In Fig. 4, an overview of the results of the self-calibration algorithm is shown: once the mounting angle are estimated, the raw data are rotated, resulting to be aligned with respect to the vehicle's axes. Differences in the accelerations are due to the forces experienced in the different locations of the vehicle. In Table I, the estimated angles are compared for the different datasets, proving that the estimated angles converge within a very narrow range.

\begin{tabular}{|c|c|c|c|c|}
\hline Location & Test \# & $\boldsymbol{\varphi}[\mathbf{d e g}]$ & $\boldsymbol{\vartheta}[\mathbf{d e g}]$ & $\boldsymbol{\psi}[\mathbf{d e g}]$ \\
\hline \multirow{3}{*}{ e-Box 1 } & 1 & -74.7930 & -23.9366 & 22.4845 \\
\cline { 2 - 5 } & 2 & -74.4650 & -23.9310 & 22.9275 \\
\cline { 2 - 5 } & 3 & -74.6607 & -23.8113 & 22.9784 \\
\hline \multirow{3}{*}{ e-Box 2 } & 1 & 4.0159 & 3.5636 & 8.9209 \\
\cline { 2 - 5 } & 2 & 3.9232 & 3.6610 & 10.1428 \\
\cline { 2 - 5 } e-Box 3 & 3 & 3.9257 & 3.6910 & 13.2746 \\
\cline { 2 - 5 } & 2 & 2.5145 & 68.9914 & -1.9422 \\
\cline { 2 - 5 } & 3 & 2.3463 & 68.8359 & -2.511 \\
\hline
\end{tabular}

TABLE I: Analysis of the estimated angles for the different boxes in all the considered tests.

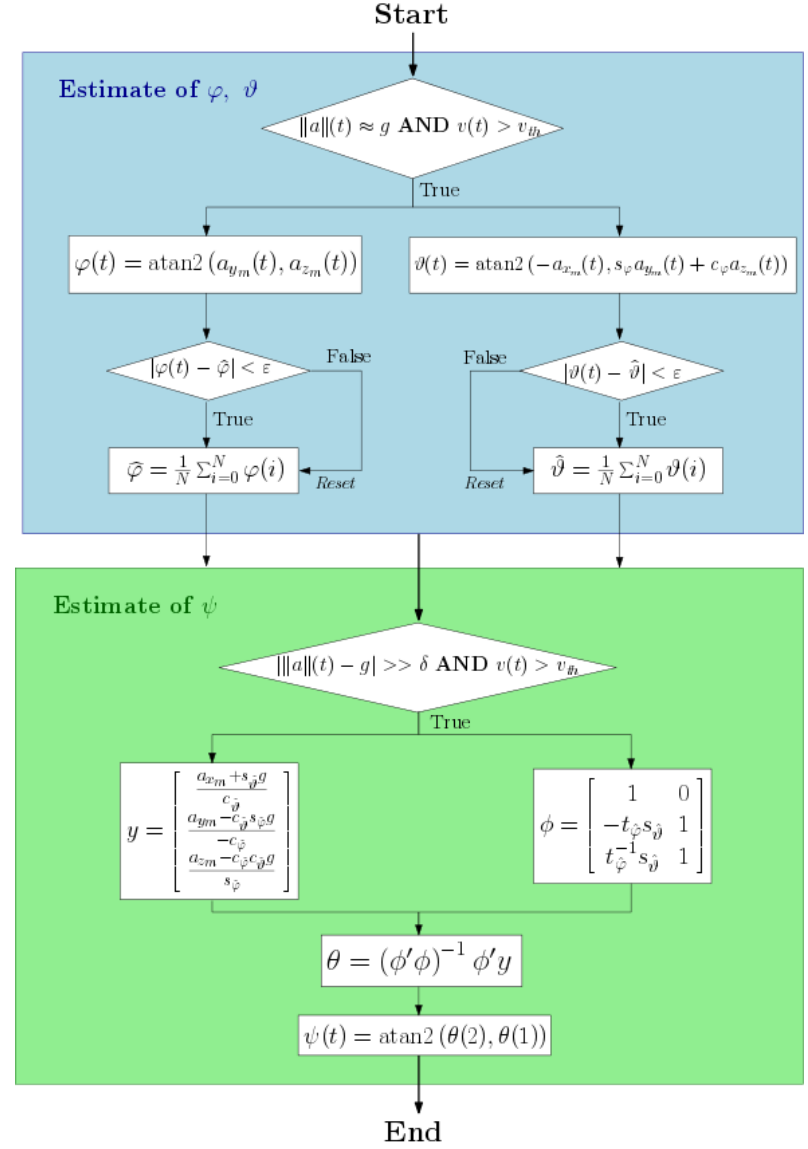

Fig. 3: The flowchart of the self-calibration algorithm.

The averaging process of $\varphi$ and $\vartheta$ plays a key role. A sensitivity analysis has been performed on the averaging window, as shown in Fig. 5. The estimate of the two angles converges in a close range even with few samples, but it settles for a window length of 4000 useful samples.

In order to study the rate of convergence in the estimate of $\psi$, the LS problem is compared with its recursive version (known as recursive least squares, RLS). As depicted in Fig. 6, RLS converges to the final estimate in 400 useful samples, $\rightarrow$ rder of magnitude smaller than the number of samp equired in the averaging process of $\varphi$ and $\vartheta$. However, it must be remarked that the estimate of $\psi$ heavily depends on data and this explains the small mismatch obtained performing the estimate with different tests.

To test the robustness of the proposed algorithm, the self-calibration algorithm is validated against different initial conditions. For all the boxes, the algorithm is run on different initial time instant, chosen randomly. This test procedure is iterated fifty times for each dataset, leading to more than a hundred tests. In Table II, the results of this test are sh As shown, the standard deviation (Std) of the angles esmonted $D$ ontained in $0.6 \mathrm{deg}$ for all the estimates, except for $\psi$. In this estimate has proved to be more sensitive to sensors' bias and drifts. This is particularly evident in one configuration, e-Box 2, in which angles $\varphi$ and $\vartheta$ are particularly small, meaning that the vertical axis is already 

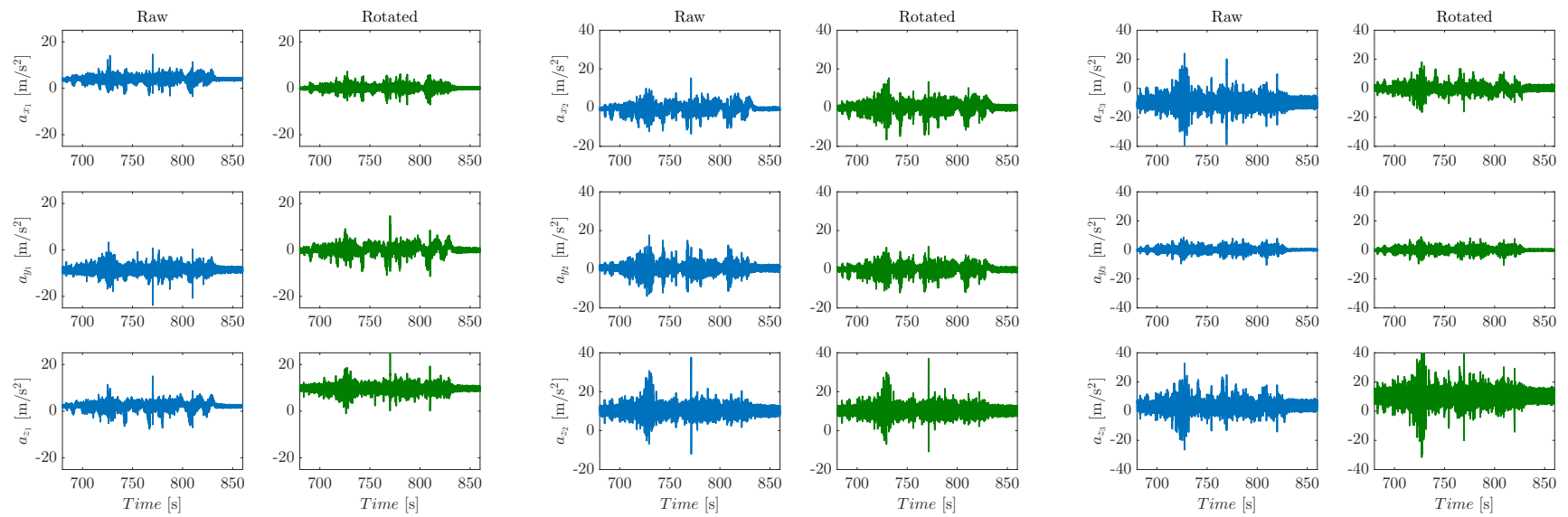

(b)

(c)

Fig. 4: An overview of the results of the self-calibration algorithm against experimental data collected by the $e$-Box 1 (Fig. 4a), e-Box 2 (Fig. 4b), and e-Box 3 (Fig. 4c): on the left, the raw acceleration vector; on the right, the accelerations after have virtually aligned the axes.
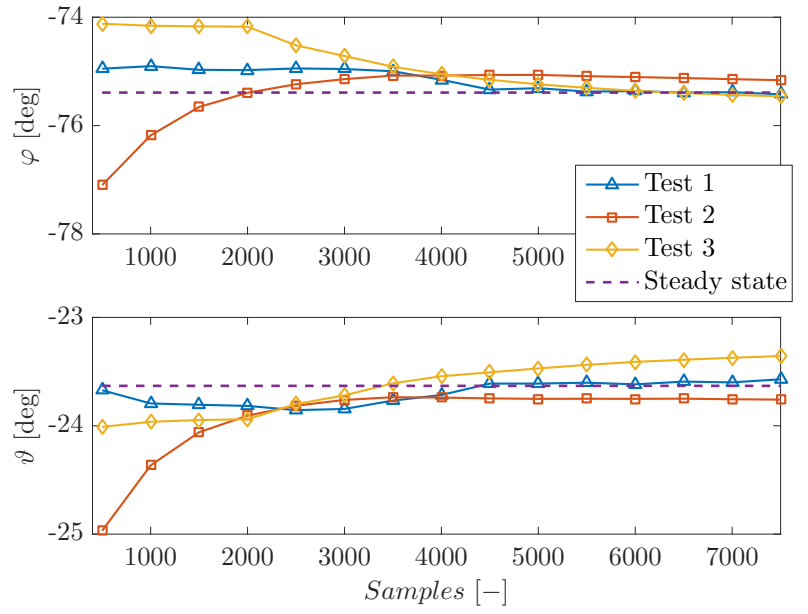

Fig. 5: An overview of the sensitivity analysis of the averaging window for the estimate of $\varphi$ and $\vartheta$. It is evident that with at least 4000 samples, the error computed in the estimate is very small with respect to the one estimated when the vehicle is standing still.

close to the vehicle's reference system. In this condition, the estimate of $\psi$ results to be more sensitive to any bias in sensors measuring $a_{x}$ and $a_{y}$.

To check the consistency of the virtually aligned axes, the rotated signals are compared by computing the crosscorrelation between the three boxes. Given two random signals $x$ and $y$, cross-correlation is a similarity metric defined as

$$
R_{x, y}(k, l)=\mathbb{E}\left[x(k) y(k+l)^{\prime}\right]
$$

where $l$ is the lag (or delay) between the two signals [13]. The cross-correlation coefficient can be normalized with respect to the autocorrelation (the correlation of the signal itself) of the two signals, leading to

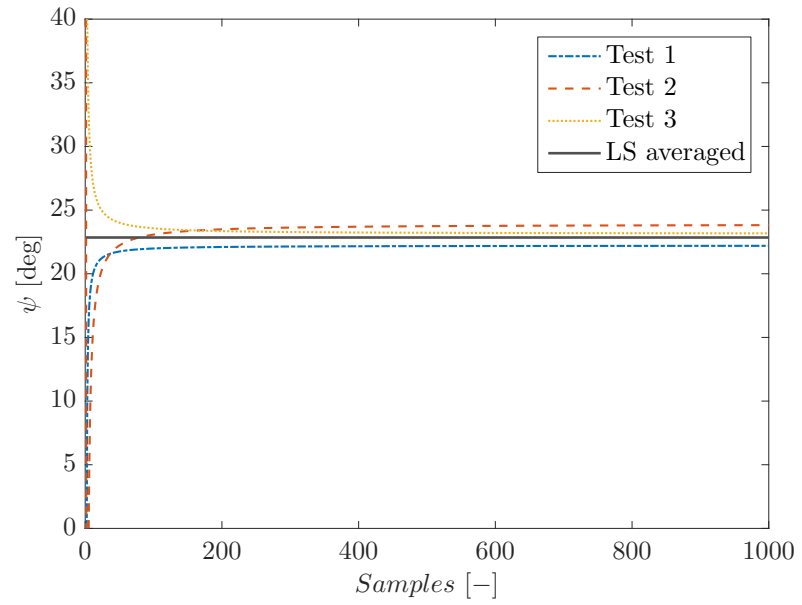

Fig. 6: The RLS convergence is compared with the averaged LS. Estimates converge in less than 400 samples. Steady state error results to be smaller than $0.92 \mathrm{deg}$.

$$
R_{x, y_{\text {norm }}}(k, l)=\frac{1}{R_{x, x}(0) R_{y, y}(0)} \mathbb{E}\left[x(k) y(k+l)^{\prime}\right],
$$

which is 0 when the two signals are orthogonal and 1 when they are completely correlated.

The normalized cross-correlation coefficient is computed on the rotated signals of all the boxes against all the test performed. Results are listed in Table III. The $z$-axis results to be perfectly aligned, with a high value of the normalized cross-correlation coefficient. Besides, the vertical axis has proved to be less excited, meaning that its estimate is less influenced by the location of the device. Axes $x-y$ show a significant correlation, though the relative location of the $e-B o x$ influences more the measured accelerations and, consequently, the correlation coefficients slightly drop. However, the mismatch is limited and the results can be considered satisfying, proving that the effectiveness of the 


\begin{tabular}{|c|c|c|c|c|c|c|c|}
\hline \multirow{2}{*}{ Location } & \multirow{2}{*}{ Test \# } & \multicolumn{2}{|c|}{$\boldsymbol{\varphi}[\mathbf{d e g}]$} & \multicolumn{2}{c|}{$\boldsymbol{\vartheta}[\mathbf{d e g}]$} & \multicolumn{2}{c|}{$\psi[\mathbf{d e g}]$} \\
\cline { 2 - 8 } & & Mean & Std & Mean & Std & Mean & Std \\
\hline \multirow{3}{*}{ e-Box 1 } & 1 & -74.5740 & 0.1472 & -24.0581 & 0.1079 & 23.3308 & 0.5159 \\
\cline { 2 - 8 } & 2 & -74.3095 & 0.1241 & -23.9478 & 0.0550 & 23.6458 & 0.5953 \\
\cline { 2 - 8 } & 3 & -74.7675 & 0.0743 & -23.7621 & 0.0362 & 23.6971 & 0.3711 \\
\hline \multirow{3}{*}{ e-Box 2 } & 1 & 3.9753 & 0.0271 & 3.5974 & 0.0641 & 8.5983 & 2.5132 \\
\cline { 2 - 8 } & 2 & 3.9398 & 0.0118 & 3.5972 & 0.0762 & 12.4725 & 3.7474 \\
\cline { 2 - 8 } e-Box 3 & 3 & 3.9151 & 0.0402 & 3.8162 & 0.0523 & 11.1468 & 2.7991 \\
\cline { 2 - 8 } & 1 & 2.3056 & 0.0781 & 68.0072 & 0.2912 & -1.8312 & 1.2517 \\
\cline { 2 - 8 } & 2 & 2.2409 & 0.2091 & 68.8345 & 0.2424 & -2.0077 & 0.8090 \\
\hline
\end{tabular}

TABLE II: Analysis of the estimated angles for all the boxes with different initial conditions. In all the cases, the the standard deviation does not exceed 0.6 deg.

proposed algorithm even in different mounting locations and orientations.

\begin{tabular}{|c|c|c|c|c|c|}
\hline \multirow{2}{*}{ Location $_{1}$} & \multirow{2}{*}{ Location $_{2}$} & \multirow{2}{*}{ Test \# } & \multicolumn{3}{|c|}{ Cross - correlation } \\
\cline { 3 - 6 } & & & $a_{x}$ & $a_{y}$ & $a_{z}$ \\
\hline \multirow{3}{*}{ e-Box 1 } & \multirow{3}{*}{ e-Box 2 } & 1 & 89.57 & 97.55 & 100 \\
\cline { 3 - 6 } & & 2 & 92.34 & 59.69 & 95.66 \\
\cline { 3 - 6 } & \multirow{3}{*}{ e-Box 3 } & 3 & 78.4 & 88.63 & 100 \\
\cline { 3 - 6 } & & 2 & 88.02 & 83.38 & 100 \\
\cline { 3 - 6 } e-Box 2 & \multirow{3}{*}{ e-Box 3 } & 1 & 95.15 & 78.7 & 100 \\
\cline { 3 - 6 } & & 2 & 84.89 & 82.43 & 100 \\
\cline { 3 - 6 } & & 3 & 77.15 & 71.68 & 100 \\
\hline
\end{tabular}

TABLE III: Correlation analysis of the rotated axes different boxes against all the considered tests.

\section{FINAL REMARKS}

This paper presented an algorithm to align the three axes of an arbitrarily mounted IMU on motorcycles. The algorithm consists in estimating three mounting angles which allow to virtually align the se axes with respect to the vehicle's reference frame. The rithm has been tested and validated against real experimental data. Results favorably prove the effectiveness of the proposed approach, limiting the amount of energy needed with respect to more classical methods due to the reduced use of GNSS system.

\section{REFERENCES}

[1] U. Maeder and M. Morari, "Attitude estimation for vehicles with partial inertial measurement," IEEE Transactions on Vehicular Technology, vol. 60, no. 4, pp. 1496-1504, 2011.

[2] Z. Syed, P. Aggarwal, X. Niu, and N. El-Sheimy, "Civilian vehicle navigation: Required alignment of the inertial sensors for acceptable navigation accuracies," IEEE Transactions on Vehicular Technology, vol. 57, no. 6, pp. 3402-3412, 2008.

[3] I. Boniolo, S. Savaresi, and M. Tanelli, "Roll angle estimation in twowheeled vehicles," IET Control Theory \& Applications, vol. 3, no. 1, pp. 20-32, 2009.

[4] Z. Syed, P. Aggarwal, C. Goodall, X. Niu, and N. El-Sheimy, "A new multi-position calibration method for mems inertial navigation systems," Measurement Science and Technology, vol. 18, no. 7, p. $1897,2007$.

[5] P. Groves, "Navigation using inertial sensors [tutorial]," IEEE Aerospace and Electronic Systems Magazine, vol. 30, no. 2, pp. 42-69, 2015.

[6] E. Vinande, P. Axelrad, and D. Akos, "Mounting-angle estimation for personal navigation devices," IEEE Transactions on Vehicular Technology, vol. 59, no. 3, pp. 1129-1138, 2010.
[7] P. D. Groves, Principles of GNSS, inertial, and multisensor integrated navigation systems. Artech house, 2013.

[8] C. De Tommasi, "Method for calibrating inertial sensor installed in arbitrary position on board vehicle and sensor system of dynamics of vehicle able to be installed on board in arbitrary position," 2014. US Patent 8,825,274.

[9] S. Savaresi and I. Boniolo, Estimate of the lean angle of motorcycles. VDM Verlag, 2010.

[10] R. C. Gonzalez and R. E. Woods, Digital image processing. Upper Saddle River, NJ: Prentice Hall, 2012.

[11] L. Sciavicco and B. Siciliano, Modelling and control of robot manipulators. Springer Science \& Business Media, 2012.

[12] A. A. Shabana, Dynamics of multibody systems. Cambridge university press, 2013.

[13] M. Verhaegen and V. Verdult, Filtering and system identification: a least squares approach. Cambridge university press, 2007. 